\title{
The organisational response of a hospital critical care service to the COVID-19 pandemic: The Groote Schuur Hospital experience
}

W L Michell, ${ }^{1,3}$ MB ChB, DA (SA), FFA (SA), Crit Care; OrcID 0000-0002-5567-897;

I A Joubert, ${ }^{1,2} \mathrm{MB}$ ChB, DA (SA), FCA (SA), Cert Crit Care; OrcID 0000-0001-8687-4224;

S Peters, ${ }^{4}$ MB ChB, BA (PPE), MMed, FCPHM; OrcID 0000-0002-5252-432;

D L Fredericks, ${ }^{1,2}$ MB ChB, FCEM (SA), Cert Crit Care (SA); OrcID 0000-0001-6663-3335;

M G A Miller, ${ }^{1,2}$ MB ChB, FCA (SA), Cert Crit Care (SA); OrcID 0000-0001-6137-7723;

J L Piercy, ${ }^{1,2}$ BSc (Hons), MB BS, FCA (SA), Cert Crit Care (SA); OrcID 0000-0003-0665-0963;

C Arnold-Day, ${ }^{1,2}$ MB BCh, MMed (Neurosurg), FCNeurosurg; OrcID 0000-0003-1748-2571;

D A Thomson, ${ }^{1,3}$ MB ChB, FCS (SA) MMed (Surg), Cert Crit Care; 0000-0003-2433-361;

R N van Zyl-Smit, ${ }^{1,5}$ MB ChB, FRCP (UK), Dip HIV (Man), MMed, FCP (SA), Cert Pulm (SA), PhD, ATSF; OrcID 0000-0002-4115-5362;

G Calligaro, ${ }^{1,5} \mathrm{MB}$ ChB, BSc (Hons), Dip PEC, FCP (SA), MMed (Int Med), Cert Pulm (SA); OrcID 0000-0001-7324-0775;

G Strathie, ${ }^{1}$ B Tech; P L Semple, ${ }^{6}$ MB ChB, FCNeurosurg (SA), MMed, PhD; OrcID 000-0002-9856-7552;

R Hofmeyr, ${ }^{7}$ MB ChB, DA, DipPEC, MMed (Anaesthesiol), FCA, FAWM; OrcID 0000-0002-9990-7459;

D Peters, ${ }^{1}$ PN; OrcID 0000-0001-7190-2894; K Dheda, ${ }^{1,5}$ MB BCh, FCP (SA), FCCP, PhD, FRCP (Lond); OrcID 0000-0001-7709-53410

\author{
${ }^{1}$ Division of Critical Care, Groote Schuur Hospital, Cape Town, South Africa \\ ${ }^{2}$ Division of Critical Care, Department of Anaesthesia and Perioperative Medicine, University of Cape Town, South Africa \\ ${ }^{3}$ Division of General Surgery, Department of Surgery, Faculty of Health Sciences, University of Cape Town, South Africa \\ ${ }^{4}$ School of Public Health \& Family Medicine, Faculty of Health Sciences, University of Cape Town, South Africa \\ ${ }^{5}$ Division of Pulmonology, Department of Medicine and UCT Lung Institute, Faculty of Health Sciences, University of Cape Town, South Africa \\ ${ }^{6}$ Division of Neurosurgery, Department of Surgery, Faculty of Health Sciences, University of Cape Town, South Africa \\ ${ }^{7}$ Department of Anaesthesia and Perioperative Medicine, Faculty of Health Sciences, University of Cape Town, South Africa
}

Corresponding author: W L Michell (lance.michell@uct.ac.za)

\begin{abstract}
Background. There are limited data about the coronavirus disease-19 (COVID-19)-related organisational responses and the challenges of expanding a critical care service in a resource-limited setting.

Objectives. To describe the ICU organisational response to the pandemic and the main outcomes of the intensive care service of a large state teaching hospital in South Africa.

Methods. Data were extracted from administrative records and a prospective patient database with ethical approval. An ICU expansion plan was developed, and resource constraints identified. A triage tool was distributed to referring wards and hospitals. Intensive care was reserved for patients who required invasive mechanical ventilation (IMV). The total number of ICU beds was increased from 25 to 54 at peak periods, with additional non-COVID ICU capacity required during the second wave. The availability of nursing staff was the main factor limiting expansion. A ward-based high flow nasal oxygen (HFNO) service reduced the need for ICU admission of patients who failed conventional oxygen therapy. A team was established to intubate and transfer patients requiring ICU admission but was only available for the first wave.

Results. We admitted 461 COVID-19 patients to the ICU over a 13-month period from 5 April 2020 to 5 May 2021 spanning two waves of admissions. The median age was 50 years and duration of ICU stay was 9 days. More than a third of the patients $(35 \% ; n=161)$ survived to hospital discharge. Conclusions. Pre-planning, leadership, teamwork, flexibility and good communication were essential elements for an effective response. A shortage of nurses was the main constraint on ICU expansion. HFNO may have reduced the requirement for ICU admission, but patients intubated after failing HFNO had a poor prognosis.
\end{abstract}

Keywords. COVID-19; intensive care; organisation; triage.

South Afr J Crit Care 2021:37(1):63-69. https://doi.org/10.7196/SAJCC.2021.v37i2.503

Contributions of the study. We describe the organisational requirements to successfully expand critical care facilities and strategies to reduce the need for invasive mechanical ventilation in COVID-19 pneumonia. We also present the intensive care outcomes of these patients in a resourceconstrained environment. 
The COVID-19 pandemic presents an unprecedented challenge to intensive care units (ICUs) around the world. Unlike a mass casualty event, the pandemic requires a medium- to long-term dynamic reorganisation of intensive care services to optimise clinical care in the face of a massive clinical burden. Although guidelines for organising an ICU response to COVID-19 are available, ${ }^{[1]}$ the response in a country with limited ICU resources will, of necessity, be different.

The Division of Critical Care at Groote Schuur hospital (GSH), the main academic hospital of the University of Cape Town, normally manages 25 ICU beds spread over the medical, surgical, cardiothoracic and source isolation ICU. Neurosurgical and cardiology ICUs are managed by their base specialities. Under normal conditions, there is a high demand for beds, and not all patients requiring intensive care can be admitted. The nurse-to-patient ratio is 1:2 and finding enough nurses to staff the units is a constant challenge. Medical staff comprises five critical care specialists, four pulmonologists, two to four fellows in critical care or pulmonology, a junior anaesthesiologist and approximately 12 rotating registrars. Eight critical care technologists and two assistant critical care technicians are responsible for ICU and theatre equipment and assist with in-hospital patient transport.

\section{Methods}

Hospital administrative records including bed occupancy rates, minutes of planning meetings, and internal communications were used to corroborate the experiences of the authors. Ethical approval to analyse anonymised clinical data from registered patient databases was obtained from the Human Research Ethics Committee of the Faculty of Health Sciences at the University of Cape Town (ref. no. HREC 244/2021).

\section{Results}

\section{Managerial response and the planning phase}

The World Health Organization (WHO) declared a global pandemic on 5 January 2020. Planning for a surge in hospital patients was initiated by the National and Western Cape Departments of Health in February 2020. The Western Cape Provincial Critical Care Forum made several recommendations. An objective ICU triage tool (http://www.sajcc.org. za/public/sup/503.pdf) based on recommendations by the Critical Care Society of Southern Africa ${ }^{[2]}$ was adopted and distributed to all emergency centres and COVID wards and referral hospitals. ${ }^{[3]}$ This included a link to the Association of Palliative Care Practitioners guidelines for managing palliative care during the COVID-19 pandemic. ${ }^{[4]}$ Transport of critically ill patients would be minimised because constraints on the ambulance service and transferring hypoxic patients on near 100\% oxygen is not feasible. A service level agreement was created to allow the transfer of appropriately triaged patients at public facilities to private hospitals, to be cared for at state expense, if no other option could be accessed. ${ }^{[5]}$

The GSH plan designated specific wards, overseen by the department of medicine, for the care of hospitalised COVID-19 patients. The wardbased service eventually expanded to 11 wards, managed by 95 doctors from various disciplines. ${ }^{[6]}$

The ICUs were designated 'COVID' or 'non-COVID' and reserved for patients requiring invasive mechanical ventilation (IMV). On 28 February 2020, the ICU consultants developed a plan to expand ICU resources. Clinical areas in the hospital were surveyed to determine how many bed spaces had the minimum support services for an ICU bed. These were: two oxygen points, two suction points and 14 electrical sockets. A total of 60 bed spaces were identified. Approximately 60 ventilators capable of supporting severe acute respiratory distress syndrome (ARDS), including loaned and 'mothballed' machines, were available. Shortages of infusion pumps, monitors and ICU beds were identified. The hospital pharmacy stockpiled essential medication. The department of anaesthesia established a team to intubate ward patients and transfer them to the ICU. ${ }^{[7]}$

Because COVID-19 ARDS is a complex illness, we aimed not to compromise on the quality of intensive care we would provide. The limit of ICU patients we could support was determined primarily by the availability of ICU staff, particularly nursing staff. Redeployment of staff was a necessity.

Initially, some clinical departments resisted the idea of becoming involved in the clinical management of what was seen as a predominantly medical and ICU condition. On 12 March 2020, the hospital's chief executive officer issued a hospital notice calling for a concerted effort from all staff: 'As health professionals and health support staff, we will aim to ensure that we continue to provide a public health service to our patients and that we support one another in doing so. Our hospital motto of 'Servamus' (we serve) and our behavioural principle of 'I will respect you and you will respect me' continue to guide us in our efforts.' Heads of departments led by example and became directly involved in the ward care of COVID-19 patients.

\section{The first wave}

The first COVID-19 ICU patient, a member of the hospital staff, was admitted on 5 April 2020. Fortunately, the national state of disaster declared on 15 March 2020 (which initially included the banning of alcohol sales and a night curfew) drastically reduced trauma admissions. ${ }^{[8]}$ With elective surgery halted, there was ICU bed capacity to accommodate the initial increase in ICU referrals

An initial six-bed 'non-COVID' ICU managed all such surgical and medical patients with overflow into the cardiothoracic ICU. A limited cardiothoracic surgery service continued throughout the year but at most times we were able to use two to four of the six beds in that unit for non-COVID ICU cases.

The situation was dynamic. Weekly consultant meetings were held to plan the next phase of the expansion. As the caseload grew, more areas were commissioned as ICUs (Figs 1 and 2). The rate of expansion depended on a varied demand for beds and the availability of resources (Fig. 2). The opening of each new area required intensive negotiations on reallocation of existing users of the area and the redeployment of medical and nursing staff (Fig. 3). The multi-disciplinary background of our consultants enabled 'boundary-spanning' negotiations between departments when 'top-down' edicts failed.

The clinical technologists took the lead commissioning new ICU areas. Equipment and disposable accessories were borrowed, repurposed or acquired on emergency purchase orders which were prioritised by the hospital finance department. Equipment and pharmaceutical companies were most supportive. Equipment to the value of ZAR3.3 million was donated by the SA Medical and Educational Foundation. The technologists worked tirelessly to clean and check ventilators and gave online tutorials to registrars and new ICU nurses on use and care of equipment.

The availability of ICU experienced professional nurses (PN) was the greatest limiting factor in expanding the ICU service. Nursing ratios were reduced from a usual $\mathrm{PN}$ to patient ratio of 1:2 to 1:3 with support from enrolled nurses and enrolled nursing auxiliaries. Nurses worked 12-hour shifts (including a one-hour break). This required $\sim 28$ nurses for each new 6-bed area. Additional nurses were drafted from the closed transplant unit, general wards, theatre recovery and out-patient department. The $33 \%$ cap on overtime hours was removed. However, the use of agency staff reduced as several of the usual ICU-experienced 


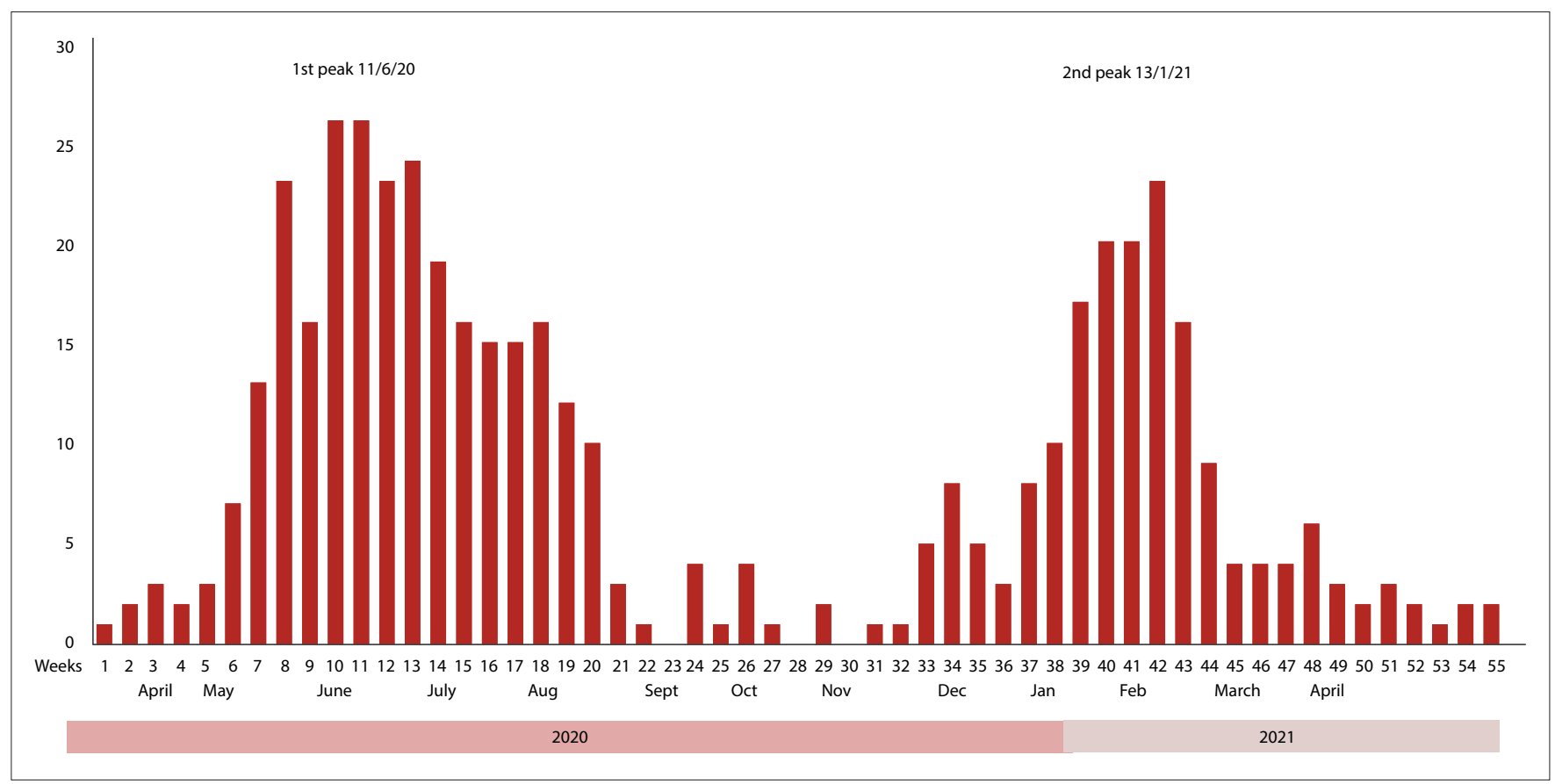

Fig. 1. Weekly COVID-19 admissions to ICU. Note the rapid rise in admissions before the peaks.

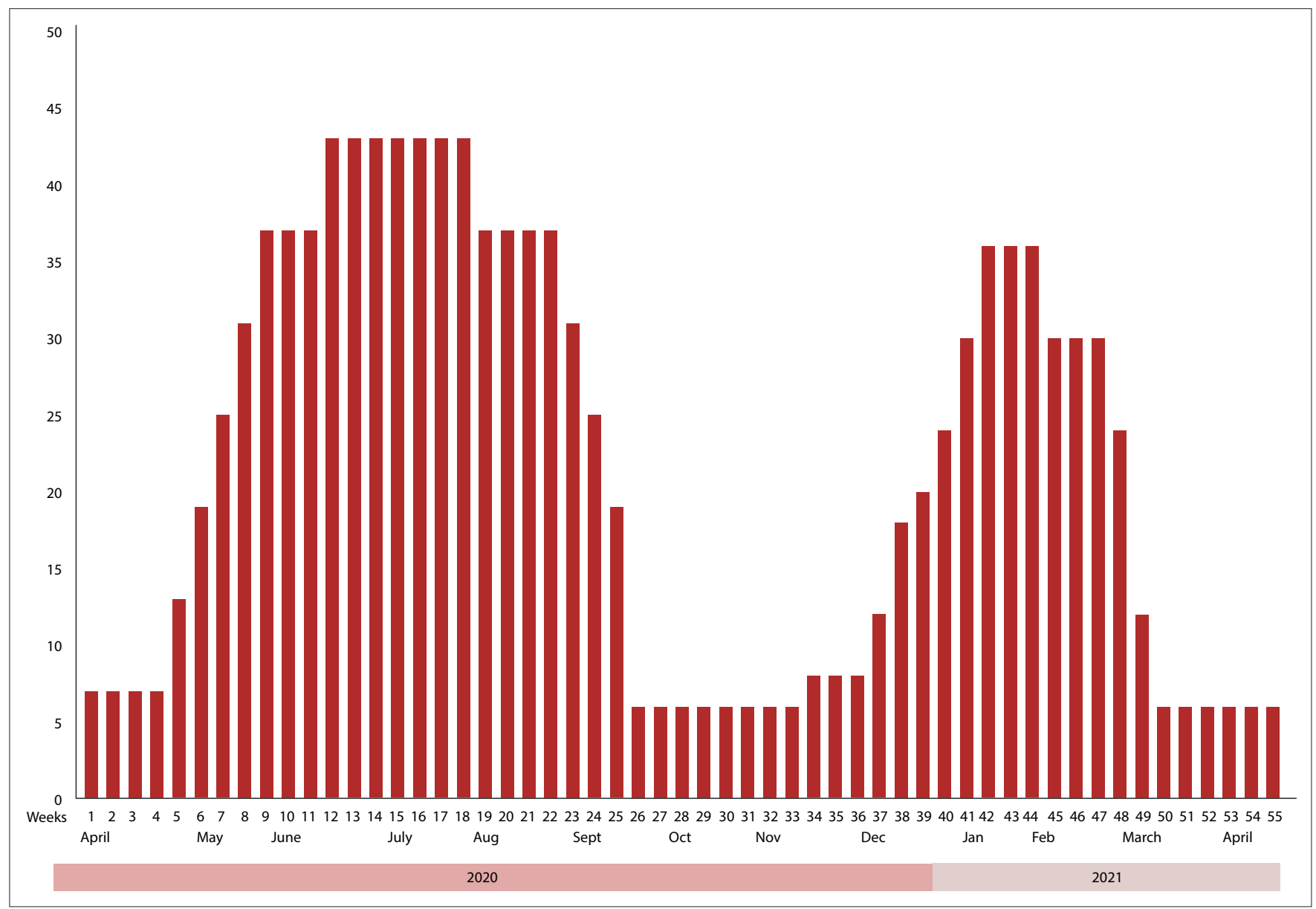

Fig. 2. Weekly COVID-19 beds in use. After the first 7 admissions, occupancy was close to 100\%. Note the prolonged bed requirements following the peaks because of prolonged ICU stays.

agency staff were lost to the field hospitals. Senior nursing managers maintained a high level of visibility in the units and participated in patient care. Unit nursing managers and the critical care clinical facilitator provided continuous in-service training.
The ICU medical staff were organised into 'firms' each managing 12 to 18 beds. At the peak of the first wave, we had three 'COVID ICU' firms and one 'non-COVID ICU' firm operating. When possible, at least one senior and one junior consultant attended the twice daily ward rounds. 


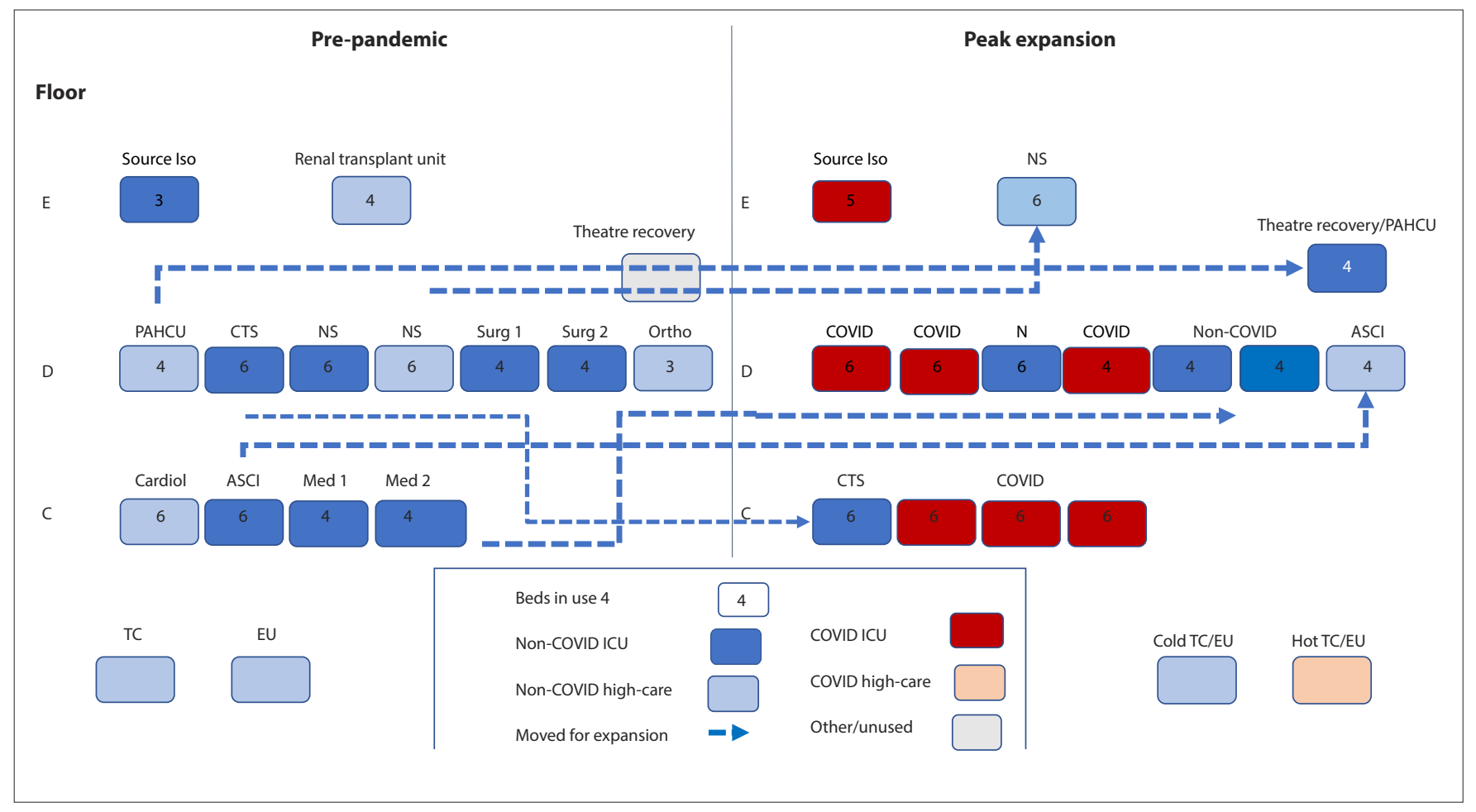

Fig. 3. Geographical expansion of ICU beds. Relocation of units to make space for COVID-19 patients. (Source Iso = source isolation unit; NS = neurosurgical; PAHCU = post-anaesthesia high care unit; CTS = cardiothoracic surgery; Surg = surgical; Med = medical; Cardiol = cardiology, TC = trauma centre; $E U=$ emergency unit). The initial 7 beds in source isolation were reduced to 5 because of nursing difficulties.

The consultants were on call for a week at a time and were responsible for making all clinical decisions and approving all admissions. During peak admission periods, the consultant taking referrals for admission triage rotated daily. They also frequently came in at night to manage clinical crises and assist junior staff.

An additional pulmonologist, neurosurgical intensivist, and anaesthesiologist, plus $\sim 18$ additional registrars were seconded to us from anaesthesia and surgery. They were organised into teams of six per firm, working shifts, with two on call during the day and one on night call. Weekends and public holidays were treated as normal workdays. Adequate time off-duty helped them cope with demanding calls. The multi-disciplinary background and seniority of some of the registrars made for strong, competent teams. Our teaching programme for registrars continued at the bedside and via on-line platforms. Two closed WhatsApp groups were used to manage medical staff logistics and were invaluable in keeping everyone informed and responding promptly to evolving problems.

\section{Staff protection}

The first few patients were managed in the 7-bed source isolation unit, which has single-bed negative pressure rooms. Once this was full, patients were cohorted in open 6-bed ICUs. Personal protective equipment in the ICU was as per WHO guidelines. Surgical masks were normally worn. Visors and N95 masks were worn for aerosolgenerating procedures. Our internal investigations revealed that all the KN95 brands available did not meet required safety standards to protect healthcare workers. ${ }^{[9]}$ Doctors often recycled N95 masks over 5 to 7 days. Reusable elastomeric respirators with replaceable filters were supplied but restricted communication to the extent that they were impractical. Clean, short-sleeved gowns were donned over personal clothes or scrub suits on entering the ICU and discarded on exiting followed by hand sanitising. Each bed area was surrounded by a 'virtual cubicle' marked on the floor. Hand sanitisation was applied, and plastic apron and gloves donned before entering the cubicle according to our normal practice, with doffing a more formal process to avoid selfcontamination. Caps and foot covers were not used. All ventilators had high-efficiency particulate air (HEPA) filters attached to their expiratory ports and active humidification was used. Ventilator disconnections and endotracheal tube cuff deflation were avoided as much as possible. All patient documentation was kept outside the virtual cubicle. Patients who tested positive for SARS-CoV-2 after admission to the non-COVID ICU were transferred to a COVID ICU as soon as practically possible.

Older staff members and those with comorbidities were designated to work in the non-COVID ICU. During the first wave, none of the ICU medical staff or intubation team contracted COVID-19, but seven (five from COVID ICUs) out of 219 ICU nurses were infected. During the second wave, 22 nurses (seven from COVID ICUs), two consultants and two registrars were infected. No clear instance of workplace exposure was identified. The technologists had a higher infection rate $(n=5 / 8)$ and may have become contaminated while removing and cleaning respiratory equipment. Fortunately, none of the ICU staff became seriously ill.

\section{Staff and family support}

It is likely that all staff members suffered emotional stress owing to the extraordinarily high mortality of our patients, and concerns for their own and their families' safety. Burnout may have been a factor contributing to the reduced availability of nursing staff during the second wave. Caring for colleagues admitted with COVID-19 pneumonia was particularly stressful. Discussing the decision to intubate with patients, telephonically discussing end-of-life decisions with families, and conveying bad news were also emotionally taxing. Weekly debriefing meetings for the doctors by a consultant psychiatrist and clinical psychologist were organised. Initially, these were virtual 


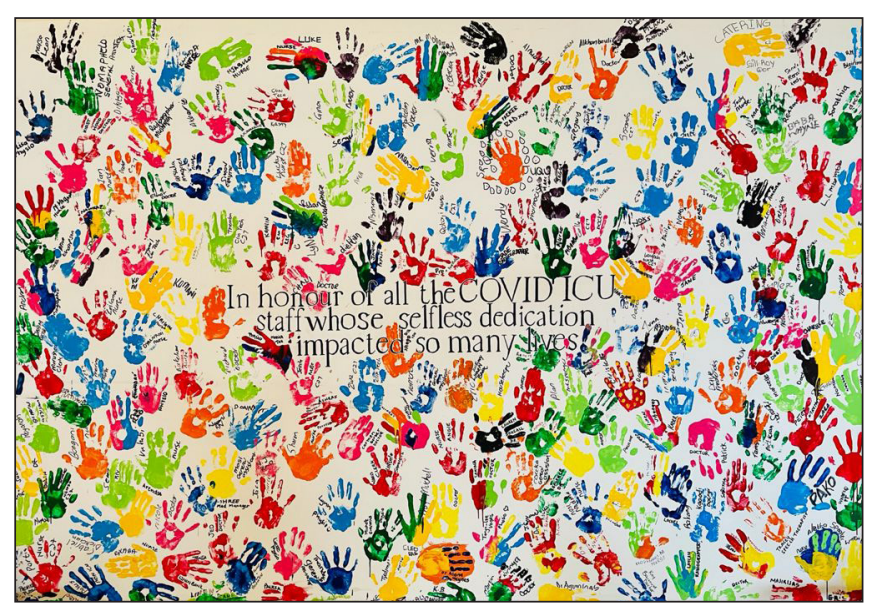

Photograph: Hand-print canvas 'The hands that cared'.

meetings, but later changed to smaller face-to-face groups. These sessions were most effective when the group was confined to people from the same peer-group, as has been suggested elsewhere. ${ }^{[10]}$ Some consultants reported that making the decision to refuse ICU admission was the worst part of the whole experience. Several registrars reported feeling overwhelmed when left alone at night with complex and dying patients. Counsellors facilitated small-group sessions for the nurses on duty and telephonic one-to-one counselling was available to all staff. Small gift packages provided to the nurses by the hospital and charities were appreciated. Informal support from a strong team spirit, banter on the chat groups and discussions during coffee breaks were very helpful.

Three mobile phones were donated by a charity to make video calls to patients' family members. The palliative care unit assisted with supporting some families and held a virtual tutorial on making telephonic calls to families and breaking bad news. A maximum of two family members were allowed to visit dying patients.

\section{Hospital oxygen supplies}

The high number of HFNO machines and ventilators in use resulted in an oxygen supply problem. During the first wave, decreases in the oxygen pipeline pressures resulted in low-pressure error messages on some ventilators, but did not affect patient care. The delivery pressure of oxygen from the hospital's vacuum-insulated evaporator was increased and had to be closely monitored. Additional oxygen storage capacity was purchased after the end of the first wave. During the second wave, GSH was using 11 to 15 tons of liquid oxygen per day, compared to a normal consumption of 1 ton per day. The Western Cape was predicted to run out of oxygen, but this was managed by halting the supply of oxygen to industry and importing oxygen from other provinces. Tanker deliveries of oxygen to the hospital had to be made daily at peak periods.

\section{The second wave}

The second wave started with several disadvantages. Western Cape suffered the highest peak incidence of cases of all the provinces ( $n=322.9 / 100000)$, almost three times higher than the first wave. ${ }^{[1]}$ The number of referrals to ICU was overwhelming. There was no preceding curfew or alcohol restriction and elective surgery had recommenced, so a greater proportion of available ICU beds accommodated non-COVID patients. The increased pressure to maintain urgent and emergency surgical services meant that the departments of surgery and anaesthesia were not able to release additional registrars early in the second wave to support ICU, and the intubation team could not be reconstituted. As the second wave expanded first in the private sector, there were less agency nurses available, as private hospitals required more nurses for their burgeoning ICUs.

\section{Clinical management}

\section{Ward care}

COVID-19-positive patients were cohorted in repurposed designated medical wards and treated with oxygen via facemask, awake selfproning, oral prednisolone, thromboprophylaxis and blood glucose control. ${ }^{[12]}$ Patients were referred to ICU if a composite assessment of respiratory effort, patient exhaustion, rising arterial partial pressure of carbon dioxide $\left(\mathrm{PaCO}_{2}\right)$ or altered mental state suggested $\mathrm{HFNO}$ failure, and if they met the ICU triage guidelines. Awake prone positioning was encouraged at every clinical encounter and reinforced by nursing staff according to clinical protocol. Most admissions to the COVID ICUs were transferred from the GSH COVID wards.

\section{High-flow nasal oxygen}

Initially, support with HFNO was not recommended because of the perceived aerosolisation risk. ${ }^{[2]}$ However, the severe shortage of ICU beds and a study that suggested that the infection risk was no more than the use of an ordinary oxygen mask ${ }^{[13]}$ prompted the use of HFNO. This expansion occurred in the wards and an improvement was immediately apparent. HFNO avoided the need to intubate some patients without requiring a higher level of nurse staffing. Patients were awake, could communicate, eat and self-prone. Patients with refractory hypoxia despite a high inspired oxygen fraction reservoir mask were trialled on HFNO. The decision to initiate HFNO was based on a protocol for the stepwise escalation of oxygen therapy and was contraindicated in patients with exhaustion or confusion. Additional machines were acquired, and HFNO high-care areas were established, facilitated by the division of pulmonology, but without any increase in nursing staff. Patients wore surgical masks, and all personnel were supplied with personal protective equipment including N95 masks and visors. Initially, only eight machines were available, but this was increased to 44 .

An observational study conducted at GSH and Tygerberg Hospital on patients with COVID-19 hypoxia showed that $47 \%$ of the patients avoided intubation or death despite a mean partial pressure of oxygen to inspired oxygen fraction ratio $\left(\mathrm{PaO}_{2} / \mathrm{FiO}_{2}\right)$ of only $76 .{ }^{[14]}$

\section{Triage}

One of the most challenging aspects of working in ICU during the COVID-19 pandemic was the prioritisation of patients referred to ICU for continued management and ventilatory support.

At the start of the first wave, reports from China, Italy and New York showed that hospital systems and ICU capacity were rapidly being overwhelmed by COVID-19 patients with severe ARDS. Overall mortality for mechanically ventilated patients was high, and survivors required prolonged ventilatory support. Reports also suggested that elderly patients, those with comorbidities and those with multiple organ failures had a higher mortality on mechanical ventilation. An objective system to triage was considered essential.

The ICU triage tool included a clear flow diagram and a calculated priority score (http://www.sajcc.org.za/public/sup/503.pdf). Scoring was based on the clinical frailty scale, the sequential organ failure assessment score (SOFA), age, and a comorbidity score. All referrals were assessed by the consultant-on-call who made the final admission decision, often in consultation with another senior colleague.

Patients were referred to ICU from within the hospital as well as from private hospitals and outlying regional and district level hospitals. The 
widespread distribution of the triage tool greatly assisted in reducing the number of unnecessary referrals to ICU.

During the first few months of the pandemic, patients who triaged as a category orange (priority 2 ) or higher were considered for ICU admission. As the bed pressure and admission numbers intensified during the first wave and second wave, only patients scoring red 1 or red 2 were considered eligible for ICU admission. Many patients were not referred to the ICU if they triaged as red 3, let alone orange or yellow priority. Ten red 1 patients, who could not be accommodated, were transferred to private hospitals.

\section{Intubation and retrieval}

During the first wave, the intubation team was dispatched to the wards and emergency unit to intubate accepted patients and transfer them to the ICU. This reduced the workload on both ward and ICU staff. Patients were only intubated once accepted for intensive care and bed availability confirmed, as there were no facilities to keep a ventilated patient waiting for an ICU bed. A standardised approach including video laryngoscopy was routinely used. ${ }^{[7]}$ Intubation was challenging, and cardiac arrests were not infrequent owing to hypoxia and hypotension. After intubation, the team transported the patient to the ICU using a portable ventilator equipped with a HEPA filter. During the first wave, the team intubated and transferred 248 patients to ICU. During the second wave, anaesthesia provided intubation skills training to medical staff in the COVID wards.

\section{Medical management in ICU}

All patients were ventilated using lung-protective strategies as far as possible. Patients were proned at least 16 hours per day when indicated. Deep sedation was maintained with propofol and morphine infusions, and muscle relaxation using a cisatracurium infusion. This was to prevent self- and ventilator-induced lung injury, and accidental disconnections and extubations. Physiotherapy was initially not available because of a perceived risk to staff but a limited service was introduced later in the year and was mainly of value in the rehabilitation phase.

Most patients received some form of corticosteroid. Dexamethasone $8 \mathrm{mg}$ daily was administered routinely after the benefit was shown in the RECOVERY trial. ${ }^{[15]}$ For thromboprophylaxis, subcutaneous enoxaparin $40 \mathrm{mg}$ was administered daily. If the D-dimer level was elevated, then full anticoagulation was considered if the bleeding risk was assessed to be acceptably low. Pantoprazole (until feeding was established), paracetamol and laxatives were routinely prescribed.

Initially, the dieticians provided telephonic advice on the enteral feed formula and dietary supplements. Later, dieticians and pharmacists joined the ICU ward round. Vitamins C and D, zinc and thiamine were prescribed for most patients. Antibiotics were only administered if a co-infection was suspected.

Secondary infections, including fungal sepsis, were common despite stringent infection prevention measures. The clinical course was often prolonged and fluctuant. Access to renal support was severely limited by the number of dialysis machines, but even when available, the outcome of patients who received it was poor. The onset of multiple organ failure was often an indication to begin end-of-life discussions with the family and most deaths followed a decision to palliate.

Tracheostomy was performed after at least 14 days of intubation and once the inspired oxygen concentration had decreased below $60 \%$. Unlike our usual practice of ICU percutaneous tracheostomy, the procedure was preferentially performed in a COVID-designated theatre by the otorhinolaryngologists because of the need for diathermy owing to anticoagulation. Survivors had a prolonged stay in ICU and required considerable physiotherapy for rehabilitation. Every transfer to the ward was celebrated by the staff and all but five discharges from ICU were discharged from the hospital alive.

\section{Extracorporeal membrane oxygenation}

Our unit has an extracorporeal membrane oxygenation (ECMO) service, and this was available for selected patients failing conventional ventilator management including proning. The challenges in providing this service related to the need for an ECMO-trained PN to be at the bedside at all times. Six COVID-19 patients failing mechanical ventilation, who had no other organ failure and were thought to have an otherwise good prognosis, received veno-venous ECMO. Three of these survived - a rate (though numbers were small) equivalent to the international experience in this selected group. ${ }^{[16]}$

\section{Clinical outcomes}

Between 5 April 2020 and 18 April 2021, the COVID ICUs admitted 461 patients. The median age was 50 (range 16 - 77) years and 50.5\% were female. The median length of ICU stay was 9 days. The median $\mathrm{P} / \mathrm{F}$ ratio on day one after being established on the ventilator was 104.5. Forty-four patients had other conditions necessitating admission to ICU and a coincidental SARS-CoV-2 infection. As of 28 April 2021, 35\% $(n=161)$ of patients survived to hospital discharge, and 4 were still in ICU. The incidental COVID-19 had a survival rate of 55\%. A detailed analysis of the outcomes of the COVID-19 ARDS patients will be reported in a separate article.

\section{Discussion}

Our unit managed a significant response to the pandemic, more than doubling the number of ICU beds when required. This was only possible because of good leadership, teamwork, effective communication and a 'whole hospital' response. The main limiting factor on our expansion was the availability of nurses. This is not a factor that can be rapidly remedied and needs to be addressed at a national level.

The organisational response of intensive care services was effective at saving the lives of many patients who failed conventional ward therapy including HFNO. Our mortality was higher than the $42 \%$ mortality shown in a recent meta-analysis of ICU outcomes. ${ }^{[17]}$ However, ICU mortality is very dependent on admission criteria. In a study of African ICUs, we reported an ICU/high care mortality of $48.2 \%$, but a mechanical ventilation mortality of $78.9 \%{ }^{[18]}$ By mainly accepting patients who had failed HFNO, we selected for a group with a poor prognosis but no other option for survival. It is also possible that persisting with HFNO worsened pulmonary damage owing to patient-induced lung injury and prolonged exposure to high inspired oxygen concentrations. Almost half the HFNO patients, who by conventional criteria required immediate intubation, ${ }^{[14]}$ survived without intubation HFNO, this intervention reducing the need for ICU admission. The reprioritising of hospital service to enable our response undoubtedly compromised the care of non-COVID patients and exposed limitations in the data available for informing the allocation of scarce healthcare resources.

As we approach a third wave, we are determined to apply the lessons we have learned. An expansion plan has been drawn up with defined triggers for reopening ICU areas proactively. Our staff are vaccinated, and we will continue to strive for excellence in patient care. 


\section{Conclusions}

Pre-planning, adaptability, leadership, teamwork, and good communication are essential in the ICU response to the COVID-19 surge and to cope with widely fluctuating longitudinal service demands. The availability of trained nurses was the main factor limiting ICU expansion. An objective triage tool ensured appropriate ICU referrals. The use of ward-based HFNO for patients who failed conventional oxygen therapy may have reduced the demand for ICU beds. An intubation and retrieval team were an effective intervention that increased safety for patients and staff. While the survival rate of ventilated COVID-19 pneumonia was poor, the ICU service provided many desperately ill patients a meaningful chance of survival.

\section{Declaration. None.}

Acknowledgements. None.

Author contributions. All authors contributed to the conception, design, results, analysis and writing of the study. All authors approved the final manuscript for submission.

Funding. None.

Conflicts of interest. None.

1. Aziz S, Arabi YM, Alhazzani W, et al. Managing ICU surge during the COVID-19 crisis: Rapid guidelines. Intensive Care Med 2020;46:1303-1325. https://doi/10.1007/s00134-020-06092-5

2. Critical Care Society of Southern Africa. Allocation of scarce critical care resources during the COVID-19 public health emergency in South Africa 05 MAY 2020 Version 3. https:// criticalcare.org.za/wp-content/uploads/2020/06/V3-2020-May-05-Allocation-of-Scarce-Criticalcriticalcare.org.za/wp-content/uploads/2020/06/N3-2020-May-05-Allocation-of-Scarce-Critical(accessed 21 April 2021)

3. Cloete K. COVID Critical Care Triage and Decision Tool. Circular H83/2020 Department of Health, Western Cape Government, 2020. https://www.westerncape.gov.za/assets/departments/health/ h83_2020_covid-19_covid_critical_care_triage_and_decision_tool.pdf (accessed 28 May 2021).

4. Palprac. Palliative care in South Africa during the COVID-19 pandemic is crucial. https://palprac. org/for-healthcare-providers/palliativecarecovid-19/ (accessed 12 May 2021)
5. Cloete K. Referral, allocation and administrative process to transfer and governance of patients from the public sector to the private sector. Circular H156/2020; Department of Health, Western Cape Government, 2020. https://www.westerncape.gov.za/assets/h1562020_covid-19_referral allocation_administration public to private.pdf (accessed 28 May 2021).

6. Mendelson M, Booysens L, Boutell A, et al. The mechanics of setting up a COVID-19 response Experiences of the COVID-19 epidemic from Groote Schuur Hospital, Cape Town, South Africa. S Afr Med J 2020;110(10):968-972. https://doi.org/10.7196/SAMJ.2020.v110i10.15215

7. Hofmeyr R, Seymour L, Peters S, Petersen A, Swanevelder J. The role of anaesthesiologists in the COVID-19 pandemic: Practical lessons from Groote Schuur experience. Updates in Anaesthesia 2021 (in press).

8. Navsaria PH, Nicol AJ, Parry CDH, Matzopoulos R, Magungo S, Gaudin R. The effect of lockdown on intentional and non-intentional injury during the COVID-19 pandemic in Cape Town, South Africa: A preliminary report. S Afr Med J 2021;111(2):110-113. https://doi. org/10.7196\%2FSAMJ.2021.v111i2.15318

9. Mottay L, le Roux J, Perumal R, Esmail A, Timm L, Sivarasu S, Dheda K. KN95 filtering facepiece respirators distributed in South Africa fail safety testing protocols. S Afr Med J 2020;111(3):234 239. https://doi.org/10.7196/samj.2021.v111i3.15381

10. Shapiro J, McDonald TB. Supporting clinicians during COVID-19 and beyond -learning from past failures and envisioning new strategies. N Engl J Med 2020;383:e142. https://doi.org/10.1056/ NEJMp2024834

11. National Institute of Communicable Diseases. An update on COVID-19 outbreak in South Africa. The first and the second wave of COVID-19 cases in South Africa. Communicable Diseases Communiqué January 2021;20(1). https://www.nicd.ac.za/wp-content/uploads/2021/01/ An-update-on-COVID-19-outbreak-in-South-Africa_The-first-and-second-wave.pdf (accessed 13 May 2021).

12. Mendelson M, Boloko L, Boutell A, et al. Clinical management of COVID-19: Experiences of Mendson M, Boloko L, Boutell A, et al. Clinical management of COVID-19: Experiences of
the COVID-19 epidemic from Groote Schuur Hospital, Cape Town, South Africa. S Afr Med J 2020;110(10):973-981. https://doi.org/10.7196/SAMI.2020.v110i10.15157

13. Li J, Fink JB, Ehrmann S. High-flow nasal cannula for COVID-19 patients: Low risk of bio-aerosol dispersion. Eur Respir J 2020;55:2000892. https://doi.org/10.1183/13993003.00892-2020

14. Calligaro GL, Lalla U, Audley G, et al. The utility of high-flow nasal oxygen for severe COVID-19 pneumonia in a resource-constrained setting: A multi-centre prospective observational study. EClin Med 2020;28(100570):1-9. https://doi.org/10.1016/j.eclinm.2020.100570

15. The Recovery Group. Dexamethasone in hospitalised patients with COVID-19. N Engl J Med 2021;384:693-704 https://doi:10.1056/NEJMoa2021436

16. Lorusso R, Combe s A, Coco V, De Piero M, Belohlavek J. ECMO for COVID-19 patients in Europe and Israel (Letter). Intensive Care Med 2021;47:344-348 https://doi.org/10.1007/s00134020-06272-3

17. Armstrong RA, Kane AD, Cook TM. Outcomes from intensive care in patients with COVID-19: A systematic review and meta-analysis of observational studies. Anaesthesia 2020;75:1340-1349. https://doi.org/10.1111/anae.15201

18. The African COVID-19 Critical Care Outcomes study (ACCCOS) investigators. Patient care and clinical outcomes for patients with COVID-19 infection admitted to African high-care or intensive care. Lancet 2021;397:1885-1894 https://doi.org/10.1016/S0140-6736(21)00441-4

Accepted 21 June 2021. 


\section{RESEARCH}

70 SAJCC August 2021, Vol. 37, No. 2 